\title{
ARTICLE
}

\section{Hollow Bio-derived Polymer Nanospheres with Ordered Mesopores for Sodium-Ion Battery}

Cite as

Nano-Micro Lett.

(2020) $12: 31$

Received: 12 November 2019

Accepted: 24 December 2019

Published online: 21 January 2020

(C) The Author(s) 2020

\author{
Yan $\mathrm{Ai}^{1}$, Yuxiu You ${ }^{2}$, Facai Wei ${ }^{1}$, Xiaolin Jiang ${ }^{1}$, Zhuolei Han ${ }^{1}$, Jing Cui ${ }^{1}$, Hao Luo ${ }^{1}$, \\ Yucen $\mathrm{Li}^{1}$, Zhixin $\mathrm{Xu}^{3}$, Shunqi Xu${ }^{4}$, Jun Yang ${ }^{3}$, Qinye Bao ${ }^{1}$, Chengbin Jing ${ }^{1}$, Jianwei Fu ${ }^{5}$, \\ Jiangong Cheng ${ }^{6}$, Shaohua Liu ${ }^{1,6} \bowtie$ \\ $\triangle$ Shaohua Liu, shliu@phy.ecnu.edu.cn \\ 1 State Key Laboratory of Precision Spectroscopy and Department of Materials, School of Physics \\ and Electronic Science, East China Normal University, Shanghai 200241, People's Republic of China \\ 2 Department of Physics and Astronomy, Shanghai Jiao Tong University, Shanghai 200240, \\ People's Republic of China \\ 3 School of Chemistry and Chemical Engineering, Shanghai Jiao Tong University, Shanghai 200240, \\ People's Republic of China \\ 4 Center for Advancing Electronics Dresden (cfaed) and Department of Chemistry and Food Chemistry, \\ Technische Universität Dresden, 01062 Dresden, Germany \\ 5 School of Materials Science and Engineering, Zhengzhou University, Zhengzhou 450052, \\ People's Republic of China \\ 6 State Key Lab of Transducer Technology, Shanghai Institute of Microsystem and Information Technology, \\ Chinese Academy of Sciences, Shanghai 200050, People's Republic of China
}

\section{HIGHLIGHTS}

- A novel coordination polymerization-driven hierarchical assembly approach for spatially controlled fabrication of phytic acid-based bio-derivatives was developed.

- The resultant ferric phytate bio-derived polymer featured hollow nanosphere architecture, ordered meso-channels, high surface area, and large pore volume, as anode material, delivering a remarkable electrochemical performance.

\begin{abstract}
Bio-inspired hierarchical self-assembly provides elegant and powerful bottom-up strategies for the creation of complex materials. However, the current self-assembly approaches for natural bio-compounds often result in materials with limited diversity and complexity in architecture as well as microstructure. Here, we develop a novel coordination polymerization-driven hierarchical assembly of micelle strategy, using phytic acid-based natural compounds as an example, for the spatially controlled fabrication of metal coordination bio-derived polymers. The resultant ferric phytate polymer nanospheres feature hollow architecture, ordered meso-channels of $\sim 12 \mathrm{~nm}$, high surface area of $401 \mathrm{~m}^{2} \mathrm{~g}^{-1}$, and large pore volume of $0.53 \mathrm{~cm}^{3} \mathrm{~g}^{-1}$. As an advanced anode material, this bio-derivative polymer delivers a remarkable reversible capacity of $540 \mathrm{mAh} \mathrm{g}^{-1}$ at $50 \mathrm{~mA} \mathrm{~g}^{-1}$, good rate capability, and cycling stability for sodium-ion batteries. This study holds great potential of the design of new complex bio-materials with supramolecular chemistry.
\end{abstract}

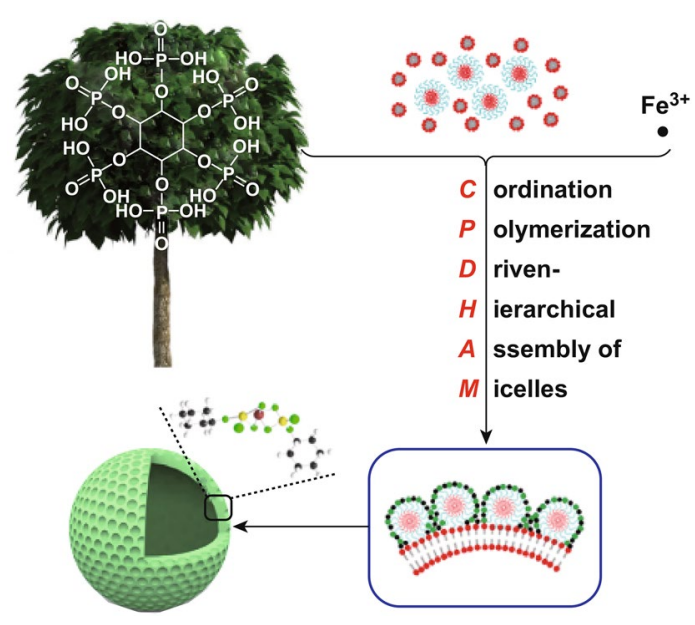

KEYWORDS Self-assembly; Biomimetic synthesis; Mesoporous polymer; Ferric phytate; Sodium-ion battery 


\section{Introduction}

Nature is a powerful designer that creates numerous wonderful works. One of the most striking masterpieces is the hierarchical structure of organism, deriving many unique properties [1, 2]. For instance, the hierarchical structure of butterfly wings can interfere and diffract with sunlight, thus presenting brilliant colors [3]. The three-layered stratified structure furnishes the shells of the Pinctada margaritifera with incredible mechanical strength, durability, and toughness [4]. The multi-scale hierarchical pore structure of trees provides channels for fast nutrient transport [5, 6]. Therefore, construction of bio-inspired materials with hierarchical structure is highly desirable for achieving various customized functions. Soft matter with abundant groups and diverse shapes, as a kind of versatile structure directing agents, is capable of manipulating the growth of discrete precursors by assembling and then instruct them to mineralize into final products with complex architecture [7, 8]. However, the build-up of hierarchies needs to ensure the homogeneity of the soft matter assemblies for the next higher levels, in that the polydispersity would be amplified and then lead to a kinetic obstacle for the further hierarchical co-assembly with the increase in size and complexity of the building blocks $[9,10]$. As a result, precisely controlled hierarchical assembly of the multiple soft matters different in size and property is still a significant bottleneck.

Recently, because of non-toxicity, easy access, wide availability, and diversity, bio-derived polymers derived from metal ion and bio-resource precursor (such as polyphenol, porphyrin, and polyacid) are emerging as attractive functional materials. In particular, as promising rechargeable electrodes materials, the inherent porous architecture of bio-derived polymer frameworks resulting from organic molecular is beneficial for hosting cations [11-13]. Furthermore, large accessible mesopores would be highly desired for their application in energy storage fields as advanced electrodes materials, because regular mesoscale nanopores would provide for materials with higher specific surface area, more exposed active sites, and specific ion transport channels [14]. Unfortunately, the synchronous control of mesoscale structure and morphology of bio-compounds from natural sources still remains unrealized so far, maybe due to the strong chelation with metal ions, continuous nucleation, and fast growth of precursors [15-17].

Here, we develop a novel coordination polymerizationdriven strategy to manipulate the hierarchical assembly of two kinds of amphiphilic soft matters for spatially controlled fabrication of ferric phytate bio-derivatives. The strong coordination among phytic acid and ferric ion promotes the discrete assemblies of pentadecafluorooctanoic acid (PFOA) and polystyrene- $b$-poly (ethylene oxide) (PS- $b$-PEO) into hollow vesicles surrounding with spherical micelles, which further confine the growth of precursors. For the first time, we have successfully achieved the synchronous control of mesoscale structure and morphology for bio-compounds. The resultant ferric phytate polymer nanomaterials are characterized by mesoporous hollow spherical architecture (hereafter termed as mFePA-HS) with a high surface area of $401 \mathrm{~m}^{2} \mathrm{~g}^{-1}$, large pore volume of $0.53 \mathrm{~cm}^{3} \mathrm{~g}^{-1}$, uniform mesoscale channels of $\sim 12 \mathrm{~nm}$. In addition, iron-based bio-resourced materials have the advantages of rich resources, environmentally friendly, low cost, good safety, and high theoretical specific capacity, so it can be used as anode or cathode in secondary batteries $[18,19]$. As a proof of concept, the bio-derivative was first explored as an advanced anode material, delivering excellent capacity, good rate capability, and cycling performance for sodium-ion batteries. This strategy has opened new avenues for spatially controlled construction of bio-based functional materials.

\section{Experimental Section}

\subsection{Chemicals and Materials}

BCP of $\mathrm{PS}_{96}-b-\mathrm{PEO}_{114}$ was synthesized in our laboratory. Phytic acid solution, Iron (III) $p$-toluenesulfonate, and pentadecafluorooctanoic acid were purchased from Maclin, Aladdin and Ark, respectively. Tetrahydrofuran and ethanol were purchased from Greagent. All chemicals were used without further purification. Deionized water was used for all experiments.

\subsection{Synthesis of mFePA-HS}

Typically, $0.05 \mathrm{~g}$ of $\mathrm{PS}_{96}-b-\mathrm{PEO}_{114} \mathrm{BCP}$ was dissolved in $1 \mathrm{~mL}$ THF. Then, $1 \mathrm{~mL} \mathrm{H}_{2} \mathrm{O}$ was added to the above solution by dropwise addition at the rate of $1 \mathrm{~min}$ one drop. After continuous stirring for half an hour, $7 \mathrm{~mL}$ of $\mathrm{H}_{2} \mathrm{O}$ was poured into the solution thus forming the micelles. After stirring, $0.016 \mathrm{~g}$ pentadecafluorooctanoic acid solution 
(1.1 wt $\%$ in ethanol) was added, followed by the addition of $0.040 \mathrm{~g}$ iron $p$-toluenesulfonate ( $11.8 \mathrm{wt} \%$ in water) and the solution turned yellow. After stirring for $4 \mathrm{~h}$, the $\mathrm{pH}$ of the synthetic medium was increased up to 3 by adding $0.140 \mathrm{~mL} 1 \mathrm{M}$ ammonia. Finally, the reaction vessel was put into an ice bath and $0.010 \mathrm{~mL}$ phytic acid solution (70 $\mathrm{wt} \%$ in water) was added into it. After continuous stirring, the color of solution slowly faded, indicating the formation of ferric phytate polymer. The pure mFePA-HS was obtained after removing the BCPs, PFOA, and excess ions by repeatedly washing with THF, ethanol, and the resulting product was further dried at $150{ }^{\circ} \mathrm{C}$ for $5 \mathrm{~h}$.

\subsection{Synthesis of Hollow Ferric Phytate Nanosphere}

First, $0.016 \mathrm{~g}$ pentadecafluorooctanoic acid solution (1.1 wt \% in ethanol) was mixed with $1 \mathrm{~mL}$ THF and $8 \mathrm{~mL}$ $\mathrm{H}_{2} \mathrm{O}$ under stirring for $30 \mathrm{~min}$, followed by the addition of $0.040 \mathrm{~g}$ iron $p$-toluenesulfonate ( $11.8 \mathrm{wt} \%$ in water). Then, the reaction vessel was put into the ice bath and $0.010 \mathrm{~mL}$ phytic acid solution (70 wt $\%$ in water) was added into it. After continuous stirring, the hollow ferric phytate was obtained after removing the PFOA, by repeatedly washing with ethanol.

\subsection{Synthesis of Blank-FePA}

First, $0.040 \mathrm{~g}$ iron $p$-toluenesulfonate (11.8 wt $\%$ in water) was added into a mixed solution of $1 \mathrm{~mL}$ THF and $8 \mathrm{~mL}$ $\mathrm{H}_{2} \mathrm{O}$. Then, after continuous stirring for $30 \mathrm{~min}, 0.010 \mathrm{~mL}$ phytic acid solution ( $70 \mathrm{wt} \%$ in water) was added into the reaction vessel. Finally, the $\mathrm{pH}$ of synthetic medium was increased up to 3 and the blank ferric phytate was obtained by centrifugation.

\subsection{Characterization and Measurements}

The morphology and structure of the mFePA-HS were investigated by scanning electron microscopy (SEM, S-4800) and transmission electron microscopy (JEM-2100F). Infrared spectra were recorded on an FTIR Spectrometer (Nicolet iS50 FTIR, Thermo). Powder XRD patterns were recorded on a Bruker X-ray diffractometer (Smartlab SE) equipped with $\mathrm{Cu}-\mathrm{Ka}$ radiation $(40 \mathrm{kV}, 20 \mathrm{~mA})$ at a rate of $10^{\circ} \mathrm{min}^{-1}$ over the range $10-80(2 \theta)$. Nitrogen absorption isotherms were measured at $77 \mathrm{~K}$ on a Quantachrome 9ASIQMUTV02UT-6. Prior to measurements, all samples were degassed in a vacuum at $120{ }^{\circ} \mathrm{C}$ for at least $12 \mathrm{~h}$. Specific surface area was determined by standard Barrett-Emmett-Teller (BET) method in the relative pressure range of 0.05-0.9 $P / P_{\mathrm{o}}$ and pore size distribution was analyzed by density functional theory (DFT). X-ray photoelectron spectroscopy (XPS) measurements were performed in a surface analysis system inducting a sample analysis chamber with the pressure of $3 \times 10^{-10} \mathrm{mbar}$, and the analyzer is Scienta-R3000. The spectra were calibrated by determining to $\mathrm{Au} 4 f_{7 / 2}$ peak position of the clean Au foil. Thermogravimetric analysis was performed on a TGA/SDTA851e instrument in an air atmosphere. Cryogenic transmission electron microscopy was performed on Tecnai F20 from FEI. Zeta-potential measurements were taken on Malvern Zetasizer Nano ZS.

\subsection{Electrochemical Measurements}

Electrochemical properties were tested by using a CR2032 coin cell. The working electrodes were prepared by mixing $70 \mathrm{wt} \%$ composite materials, $20 \mathrm{wt} \%$ carbon black as a conductive agent and $10 \mathrm{wt} \%$ polyvinylidene difluoride (PVDF) as binder. After coating the slurry on a $\mathrm{Cu}$ foil, the electrodes were dried at $80^{\circ} \mathrm{C}$ for $6 \mathrm{~h}$ and then transferred to a vacuum oven at $120^{\circ} \mathrm{C}$ for $12 \mathrm{~h}$. The loading amount of the electrode was kept at $\sim 1.5 \mathrm{mg} \mathrm{cm}^{-2}$. The assembly of all coin cells was conducted in an argon-filled glovebox, sodium metal was used as the counter and reference electrode, $1 \mathrm{M}$ $\mathrm{NaClO}_{4}$ in the mixture of EC/PC (1:1) was used as electrolyte, and glass microfiber filters from Whatman were used as separator. Electrochemical tests were conducted by a Land battery system; the voltage window was $0.001-3.0 \mathrm{~V}$. Cyclic voltammetry (CV) tests were carried out by using the threeelectrode customized cell. The $\mathrm{CV}$ measurements were taken on a CHI760E electrochemical workstation at a scan rate from 0.1 to $10 \mathrm{mV} \mathrm{s}^{-1}$.

\section{Results and Discussion}

SEM images reveal the well-defined spherical morphology of the resultant ferric phytate polymer nanomaterial. As shown in Fig. 1a-c, the ordered mesopores are uniformly 
distributed on the surface of the nanosphere, and the inserted picture in Fig. 1a indicates that the phytic acid comes from biology. The pore size and wall thickness of mesopores are $\sim 12$ and $\sim 10 \mathrm{~nm}$, respectively. The size distribution of the particles was measured by dynamic light scattering (DLS), showing the average particle size of $\sim 450 \mathrm{~nm}$ (Fig. 1d). By contrast, only irregular ferric phytate nanoparticles were obtained at the absence of block co-polymer (BCP) PS$b$-PEO and PFOA (blank-FePA) under the same conditions.

Interestingly, TEM images show the hollow structure of the ferric phytate nanospheres, and the thickness of the shells is approximately from 15 to $55 \mathrm{~nm}$ (Fig. S2). Furthermore, the morphology of mFePA-HS varies with the thickness of the shell, which exhibits a regular hollow structure with ordered mesopores when the shell is thick enough (Fig. 2). Otherwise, it appears in vesicles with mesopores uniformly distributed on the surface. The possible reason lies in the difficulty to support a spherical skeleton when the shell of mFePA-HS is too thin $[20,21]$. The integrated energy-dispersive X-ray spectroscopy (EDS) analysis elemental mapping images show that $\mathrm{C}, \mathrm{O}, \mathrm{P}$, and Fe elements are uniformly distributed in the hollow spheres (Fig. 2c), and the atomic composition of $\mathrm{C}, \mathrm{O}, \mathrm{P}$, and Fe elements is approximately $32.33 \%, 38.20 \%, 12.69 \%$, and $16.79 \%$, respectively (Fig. S3). The relative mole ratio of $\mathrm{Fe} / \mathrm{P}$ in mFePA-HS is calculated to be $0.73 / 1$. The inserted selectedarea electron diffraction pattern of mFePA-HS shows no regular lattices, indicating the amorphous frameworks of mFePA-HS (Fig. 2b).

To further survey the pore structure of mFePA-HS, the Brunauer-Emmett-Teller (BET) measurements were taken. The $\mathrm{N}_{2}$ adsorption-desorption isotherms of mFePA-HS can be categorized as type IV with a $\mathrm{H} 3$ hysteresis loop (Fig. 3a). The appearance of a hysteresis loop $\left(P / P_{\mathrm{o}}\right.$ of 0.4-0.9) confirms the presence of mesopores caused by the spherical PS- $b$-PEO micelles. Additionally, the BET surface area of the mFePA-HS is $401 \mathrm{~m}^{2} \mathrm{~g}^{-1}$, much higher than that of blank-FePA $\left(108 \mathrm{~m}^{2} \mathrm{~g}^{-1}\right)$ (Table 1). Fourier-transform
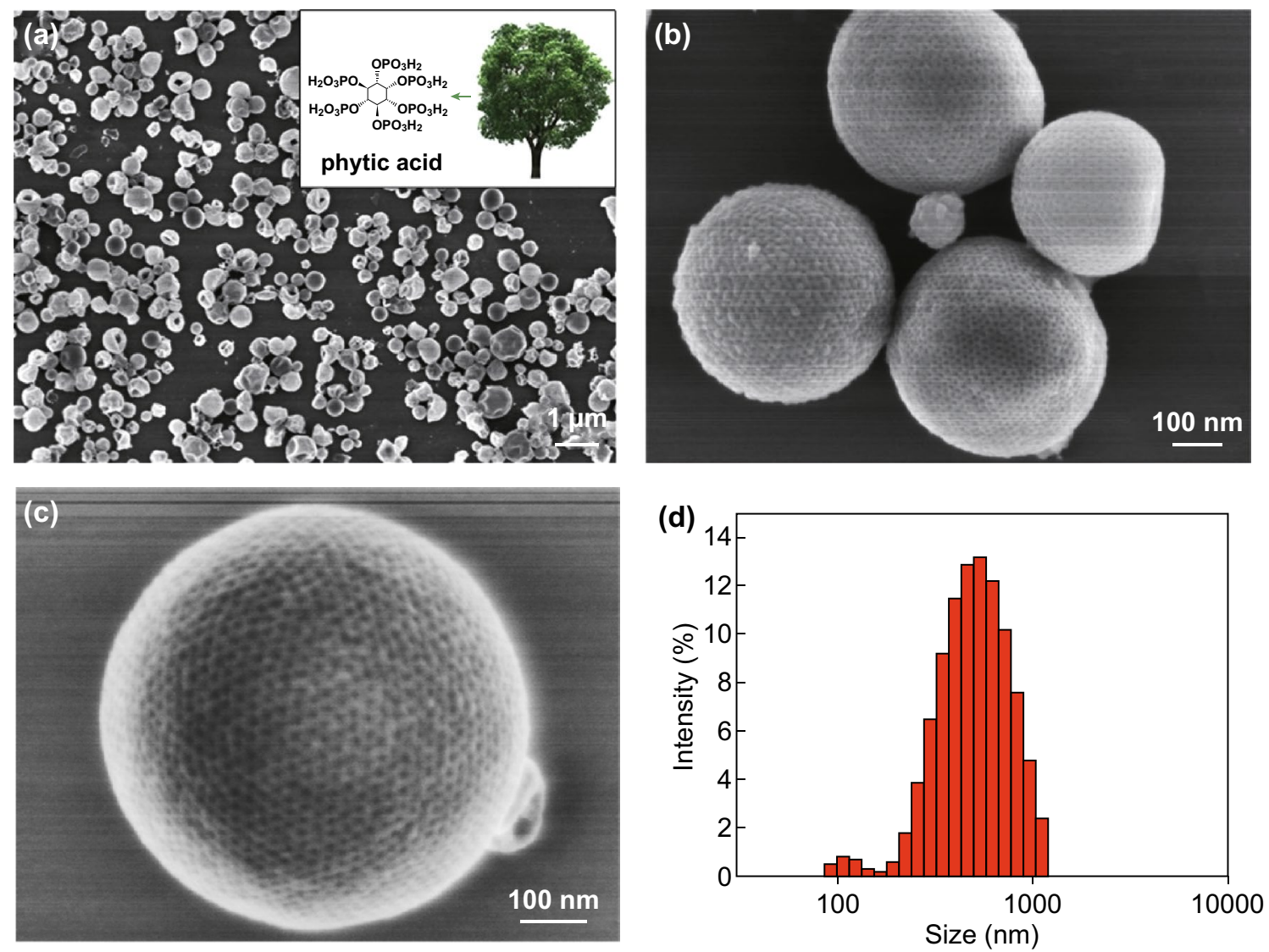

Fig. 1 Morphology of mFePA-HS. a-c SEM images of mFePA-HS at different magnifications. d The particle size distribution of mFePA-HS measured by dynamic light scattering 

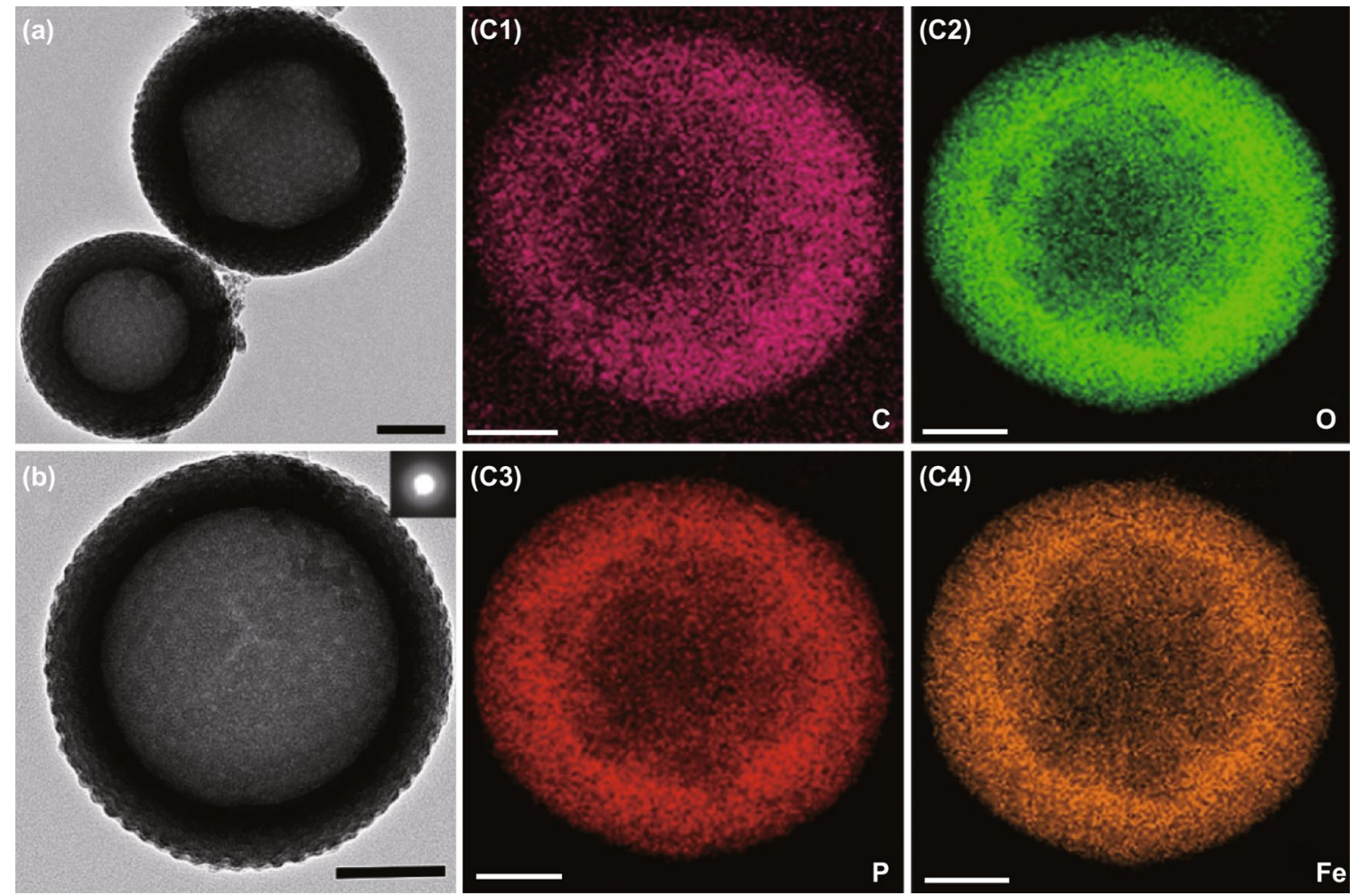

Fig. 2 Structure of mFePA-HS. a, b TEM images. c EDX element mappings of mFePA-HS (scale bar $100 \mathrm{~nm}$ )

infrared (FTIR) spectra were also performed to figure out the polymeric framework of materials and evaluate the removal of the templates (Fig. 3b). The peak at $530 \mathrm{~cm}^{-1}$ can be ascribed to the stretching vibration of $\mathrm{Fe}-\mathrm{O}$; the strong band at $1060 \mathrm{~cm}^{-1}$ is associated with the tetrahedral stretching vibration of $-\mathrm{CPO}_{3}$ group [22]. Meanwhile, the peaks at 1400 and $1640 \mathrm{~cm}^{-1}$ are corresponding to the $\mathrm{P}-\mathrm{C}$ stretching vibration and the $-\mathrm{CH}-$ bending stretching vibration, respectively [13]. In addition, ranging from 500 to $4000 \mathrm{~cm}^{-1}$, mFePA-HS exhibits almost the same FTIR signals with blank-FePA, indicating no characteristic peaks of PS- $b$-PEO in the resultant mFePA-HS, as well as -COOH of PFOA. The above results confirm the complete removal of templates as well as the formation of the ferric phytate.

The surface properties of mFePA-HS and blank-FePA were evaluated by XPS. The XPS spectrum of Fe $2 p$ shows two main peaks at 725.9 and $712.2 \mathrm{eV}$, which can be assigned to $\mathrm{Fe} 2 p_{1 / 2}$ and $\mathrm{Fe} 2 p_{3 / 2}$ electron bonding energy, respectively. Furthermore, the shake-up peaks at the binding energy of $725.9 \mathrm{eV}$ manifest the +3 valence state of $\mathrm{Fe}^{3+}$ (Fig. 3c) [15]. Figure $3 \mathrm{~d}$ shows the spectrum of $\mathrm{P} 2 p$; the intense peak located at $133.6 \mathrm{eV}$ can be assigned to the $\mathrm{P} 2 p$ energy level of pentavalent phosphorus [23]. Powder X-ray (PXRD) diffraction patterns of mFePA-HS and blank-FePA (Fig. S5) give no signatures because of their amorphous structure, consistent with the TEM result (Fig. 2b).

To investigate the component of mFePA-HS, we further conducted a thermogravimetric analysis (TGA) of mFePAHS with a heating rate of $10{ }^{\circ} \mathrm{C} \mathrm{min}-1$ under a continuous air flow. The TGA profile shows two stages of weight loss: The first one (20.5 wt \%) up to $200^{\circ} \mathrm{C}$ is ascribed to the loss of adsorbed water (Fig. S6); and the second one (34.3 wt\%) from 200 to $500{ }^{\circ} \mathrm{C}$ corresponds to the burning of organics from the framework [13]. Again, it proves that the resultant coordination polymer is composed of organic ligands.

In order to further clarify the growth mechanism of mFePA-HS, we conducted a series of the controlled experiments. As mentioned above, under the same conditions, only irregular particles of Fe-PA can be obtained without using BCP and PFOA templates (Fig. S7). Similarly, solo BCP also led to irregular particles (Fig. S8). However, hollow FePA nanospheres (PFOA-Fe-phytic acid system) can be 

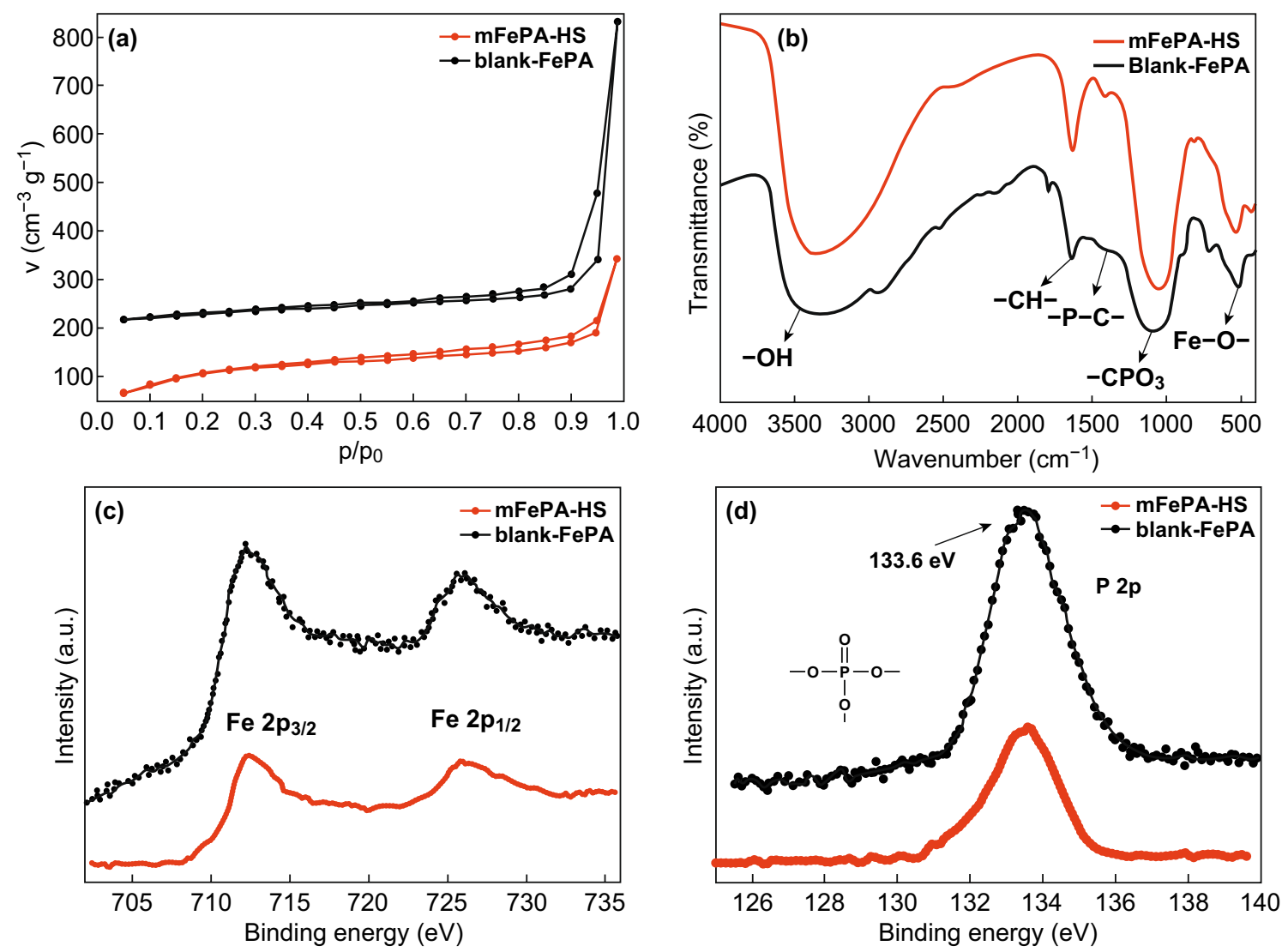

Fig. 3 Characterization of mFePA-HS. a $\mathrm{N}_{2}$ adsorption-desorption isotherms of mFePA-HS and blank-FePA; the curve of blank-FePA has been moved up for $200 \mathrm{~cm}^{-3} \mathrm{~g}^{-1}$. b FTIR spectra of the mFePA-HS and blank-FePA.c XPS spectra of Fe $2 p$. d XPS spectra of P $2 p$

Table 1 Porous properties of the mFePA-HS and blank-FePA

\begin{tabular}{llll}
\hline Samples & $\begin{array}{l}\text { Surface area } \\
\left(\mathrm{m}^{2} \mathrm{~g}^{-1}\right)\end{array}$ & $\begin{array}{l}\text { Pore volume } \\
\left(\mathrm{cm}^{3} \mathrm{~g}^{-1}\right)\end{array}$ & $\begin{array}{l}\text { mesopore } \\
\text { size }^{\mathrm{b}}(\mathrm{nm})\end{array}$ \\
\hline mFePA-HS & 401 & 0.53 & 12 \\
blank-FePA & 108 & 0.39 & - \\
\hline
\end{tabular}

${ }^{\text {a }}$ Surface area was obtained based on the Brunauer-Emmett-Teller (BET) method

${ }^{\mathrm{b}}$ The mesopore size was obtained from SEM characterization and averaged at least 50 points

generated in the use of PFOA only (Fig. S9). The proposed growth mechanism for the hollow FePA nanospheres is illustrated in Fig. 4a.

As is known, PFOA, a kind of aliphatic fluorosurfactant, can form spherical micelle with a diameter of less than $3 \mathrm{~nm}$ once its concentration exceeds critical micelle concentration (CMC) (Fig. 4a-1) [24, 25]. When ferric ions were added into the PFOA solution, they would be absorbed on the surface of the micelle of PFOA due to the electrostatic interaction (Fig. 4a-2). Nonetheless, no obvious assemblies were detected in their solution, as shown by cryogenic transmission electron microscopy (Cryo-TEM), indicating the absence of vesicle in this procedure. Upon the addition of phytic acid, the hollow ferric phytate nanospheres appeared, possibly because that the stronger coordination polymerization interactions between phytic acid and ferric ion induced the rearrangement and reassembly of PFOA into a larger vesicle (Fig. 4a-3) [26].

In the system of mFePA-HS, after the addition of PS$b$-PEO into the mixed solution of $\mathrm{THF} / \mathrm{H}_{2} \mathrm{O}$, the monodispersed spherical micelles of PS- $b$-PEO with a diameter of $12 \mathrm{~nm}$ formed, clearly evidenced by Cryo-TEM (Fig. S10). Subsequently, PFOA ethanol solution was added into BCP solution, the assemblies of PFOA and BCP micelles attract each other to form a hierarchical superstructure by hydrogen bonding (Fig. 4b-2) [27]. This process can be studied by the surface potential change in solution, which decreases from 
(a)

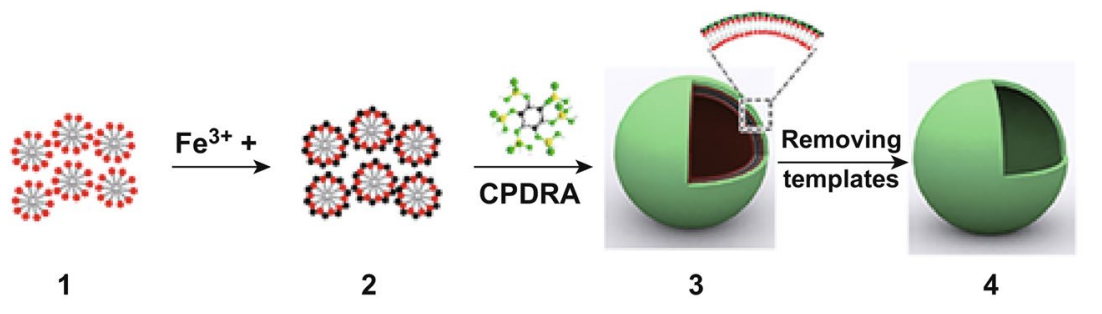

(b)

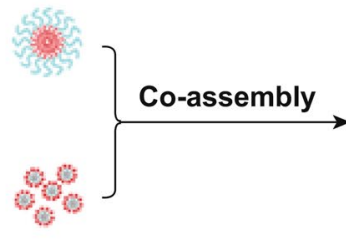

1

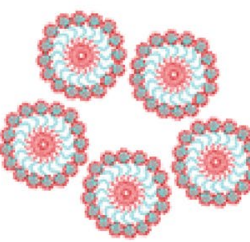

2

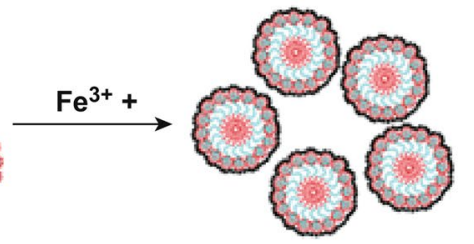

3

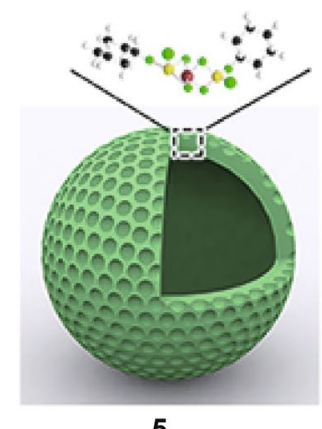

5

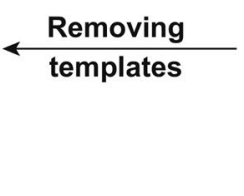

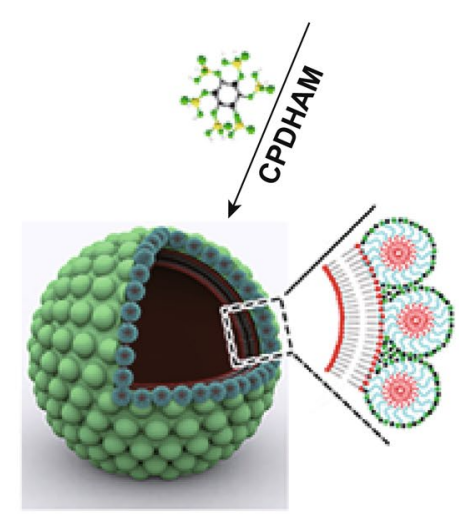

4

Fig. 4 Scheme of the synthesis of hollow FePA nanospheres (a) and mFePA-HS (b). a: (1) The spherical micelles assembled by PFOA. (2) Ferric ions were absorbed on the surface of PFOA micelles due to electrostatic interaction. (3) The formation of pristine ferric phytic acid hollow sphere with the addition of phytic acid by the method of coordination polymerization-driven reassembly (CPDRA) of PFOA micelles. (4) The resultant ferric phytic acid hollow sphere. b: (1) The preformed spherical micelles of BCP and PFOA. (2) Hierarchical co-assembly of PFOA and BCP micelles. (3) Ferric ions were absorbed on the surface of PFOA@BCP. (4) The formation of pristine mesoporous ferric phytate hollow sphere with the addition of phytic acid by coordination polymerization-driven hierarchical assembly of micelles (CPDHAM). (5) The obtained mesoporous ferric phytate hollow sphere after the template removal

-6.4 to $-13.1 \mathrm{mV}$ after the addition of PFOA into BCP solution, because of their strong interaction (Figs. S11 and S12). However, Cryo-TEM images do not show changes for the mixed micelles of PFOA and BCP(PFOA@BCP, Fig. S13), possibly due to the lower contrast and smaller size of PFOA micelles. Furthermore, after the positively charged ferric ion was added into the system, the zeta potential of the solution increased up to $-2.61 \mathrm{mV}$. In this scenario, ferric ion was strongly attracted to the surface of PFOA@ BCP assemblies (denoted as Fe@PFOA@BCP, Fig. 4b3) by the electrostatic interaction between ferric ions and PFOA (Fig. S14). Similarly, Cryo-TEM reveals the spherical morphology of the Fe@PFOA@BCP co-assemblies, without showing visual changes after the addition of ferric ions (Figs. 4b-3 and S15).

However, according to our observation, when the phytic acid molecules were added into the Fe@PFOA@BCP coassembled solution, the mesoporous FePA hollow nanospheres (mFePA-HS) appeared. In contrast, the PFOAFe-phytic acid system also generated the hollow FePA nanospheres, independent of the use of BCP. It means that the intervention of $\mathrm{BCP}$ could not interfere with the assembly of PFOA into vesicles. Even at the presence of BCP micelles, the phytic acid molecules can still chelate with 
ferric ions, which actuate PFOA molecules to rearrange into hollow vesicles (Fig. 4b-4). Moreover, the introduction of BCP endows mFePA-HS with regular mesopores, because BCP micelles could uniformly self-assemble on the surface of the vesicles with the assistance of $\mathrm{H}$-bonding interaction within BCP and PFOA (Fig. 4b-4, b-5). Furthermore, we did a controlled experiment by using octanoic acid as a substitute for PFOA to explore whether the PFOA can be replaced by carboxylic acid surfactants, which possess similar molecular structure. However, as the SEM images show, only hollow ferric phytate nanosphere constructed by irregular particles with no mesoporous can be obtained (Fig. S16). This may be ascribed to the unique hydrophobic fluoroalkyl structure of PFOA [28, 29].The exact mechanism for the growth of mFePA-HS awaits further clarification.

We further evaluated the electrochemical performances of mFePA-HS, as a new type of electrode material for the sodium-ion storage. Figure 5a shows the cyclic voltammetry (CV) curves of mFePA-HS at a low scan rate of $0.1 \mathrm{mV} \mathrm{s}^{-1}$. In the first cycle, there is an irreversible peak at $\sim 0.56 \mathrm{~V}$, which can be ascribed to the formation of solid electrolyte interphase (SEI) layers, decomposition of electrolyte, and the irreversible sodiation reaction of mFePA-HS [30].
However, in the subsequent cycles, this peak vanishes and the $\mathrm{CV}$ curves almost overlap due to the formation of a stable SEI. Furthermore, CV curves of mFePA-HS at different scan rates were obtained, obvious peaks can be observed at cathodic and anodic scans, respectively (Fig. 5b) [31].

Figure $5 \mathrm{c}$ depicts the discharge and charge curves after the initial cycle at a current density of $50 \mathrm{~mA} \mathrm{~g}^{-1}$ [32]. The mFePA-HS exhibits a remarkable discharge capacity of $540 \mathrm{mAh} \mathrm{g}^{-1}$ in the second cycle, indicating great capability of Na storage [33, 34]. In contrast, the blank-FePA exhibits an inferior electrochemical performance with a lower capacity of $340 \mathrm{mAh} \mathrm{g}^{-1}$. The higher capacity of mFePA-HS can be attributed to the unique porous structure, which is beneficial to the sufficient contact with the electrolyte as well as the rapid transport of sodium ions $[35,36]$. An obvious plateau can be observed at about $1.1 \mathrm{~V}$ in all cycles for the discharging curves. In addition, the discharge/charge profiles almost overlap during the cycles, indicating a good structural stability of the mFePA-HS electrode.

The rate capability of mFePA-HS was evaluated at various current rates from 50 to $500 \mathrm{~mA} \mathrm{~g}^{-1}$. As shown in Fig. $5 \mathrm{~d}$, the electrode of mFePA-HS delivers a reversible capacity
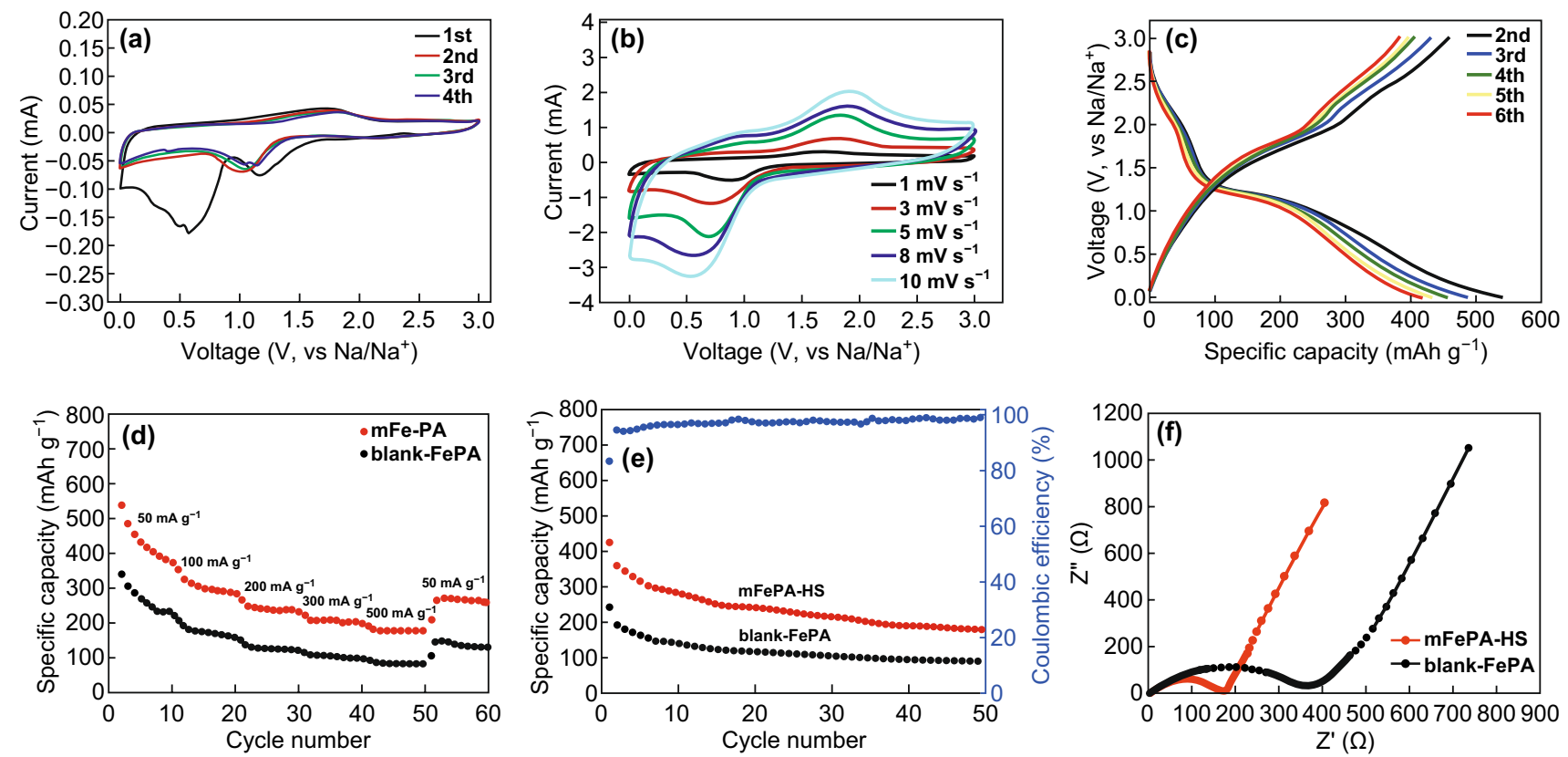

Fig. 5 The electrochemical performance of mFePA-HS and blank-FePA. a Cyclic voltammetry (CV) curves of mFePA-HS. b CV curves of mFePA-HS at different scan rates. $\mathbf{c}$ The discharge and charge curves after the initial cycle of mFePA-HS. d The rate capability of mFePA-HS and blank-FePA. e The cycling performance of mFePA-HS and blank-FePA at a current density of $200 \mathrm{~mA} \mathrm{~g}^{-1}$. f Nyquist plots of mFePA-HS and blank-FePA at room temperature 
of $400,300,240,200$, and $180 \mathrm{mAh} \mathrm{g}^{-1}$ at a current of $50,100,200,300$, and $500 \mathrm{~mA} \mathrm{~g}^{-1}$, respectively. Contrastively, the blank-FePA exhibits a much lower rate capability at the same current rates. After changing the current density to $50 \mathrm{~mA} \mathrm{~g}^{-1}$, a reversible capacity of $280 \mathrm{mAh} \mathrm{g}^{-1}$ remains, manifesting the good rate capability of mFePAHS. Figure 5e displays the cycling performance of the electrodes of mFePA-HS and blank-FePA at a current density of $200 \mathrm{~mA} \mathrm{~g}^{-1}$, which were activated at $50 \mathrm{~mA} \mathrm{~g}^{-1}$ in the initial cycle. After 50 cycles, the electrode of mFePA-HS remains at a capacity of $180 \mathrm{mAh} \mathrm{g}^{-1}$, far higher than that of blank-FePA ( $85 \mathrm{mAh} \mathrm{g}^{-1}$ ). In addition, we also evaluated the performance of the mFePA-HS after 500 cycles, the capacity remains $134 \mathrm{mAh} \mathrm{g}^{-1}$, which is much better than that of blank-FePA (44 $\mathrm{mAh} \mathrm{g}^{-1}$ ) (Fig. S18). Remarkably, although the capacity of mFePA-HS decreases during the cycles, the Coulombic efficiency still approaches about $98 \%$. However, it is worth noting that the cycle stability is also strongly dependent on the compatible electrolyte systems, which should be further investigated.

The effects of the hollow mesoporous structure toward improving the sodium storage performance of the compound were investigated byEIS analysis. Figure $5 f$ presents the Nyquist plots of mFePA-HS and blank-FePA at room temperature, both of which comprise a depressed semicircle in the moderate-frequency area and an inclined line in the low-frequency region [37]. The medium-frequency semicircle normally can be ascribed to the interfacial charge-transfer resistance $\left(R_{\mathrm{ct}}\right)$; the obviously smaller diameter of the medium-frequency semicircle of our mFePA-HS electrode reveals its lower $R_{\mathrm{ct}}$ than that of the blank-FePA, reflecting easier charge-transfer reaction in the current battery system [38]. Furthermore, the low-frequency inclined line is linked to the Warburg impedance $\left(Z_{\mathrm{w}}\right)$, which can be interpreted as the diffusion of sodium ion in the host solid-state phase [39].

Finally, SEM images of electrodes before and after cycling were obtained. According to the SEM observation, there are some structural damages in both samples, which may be ascribed to the strong volume change in the electrode. Moreover, comparing the SEM images of blank-FePA electrode before and after cycling, it can be observed that the irregular particles are divided into smaller active particles, indicating the pulverization of the material (Fig. S19) [40]. As known, large volume expansion leads to the pulverization and agglomeration of particles, which could be observed in the SEM images. Furthermore, it brings about the instability of solid electrolyte interface membrane, resulting in the reduction in cyclic stability [41]. In some degree, the problem may be solved by the introduction of volume expansion buffer materials or the construction of microstructures.

The above electrochemical characterization adequately demonstrates that mFePA-HS exhibits superior performance, which is related to the peculiar structure of itself. The open mesopores facilitate the infiltration of electrolyte and significantly shorten the diffusion distance of sodium ions in the solid phase [42]. Additionally, the high surface area would afford more electrochemical reaction interfaces, thus improving the rate capability [35]. Besides, the hollow architecture accommodates volume expansion during the insertion/extraction of sodium [43]. The combination of these features endows the mFePA-HS electrode with the outstanding capacity and reversible electrochemical reaction.

\section{Conclusions}

In summary, we have developed a new coordination polymerization-driven hierarchical assembly of micelle approach for the fabrication of hollow bio-derivatives with ordered mesopores. Driven by the strong coordination between phytic acid and ferric ion, the discrete amphiphilic micelles of PFOA and BCP further rearrange into the hollow vesicles surrounded by the spherical BCP micelles. This procedure accompanies with the confined growth of ferric phytate, enabling the formation of hollow mesoporous ferric phytate. Furthermore, considering other precursors that have similar groups with phytic acid, this method may be appropriate for other acids, such as phosphoric acid, 1-hydroxyethane1,1-diphosphonic acid, etc.

For the first time, the synchronous control on morphology and mesoscale structure for bio-compounds enriches the material with a large surface area of $401 \mathrm{~m}^{2} \mathrm{~g}^{-1}$, abundant pore volume of $0.53 \mathrm{~cm}^{3} \mathrm{~g}^{-1}$, and regular mesoporous channels of $\sim 12 \mathrm{~nm}$. As an unprecedented anode material for sodium-ion batteries, such hollow mesoporous bio-derivative nanospheres exhibit remarkable electrochemical performance. Given the similar coordination ability of the other bio-compounds with metal ions, our study would provide a feasible paradigm for spatially controlled construction of a series of bio-based functional materials. 
Acknowledgements This work was financially supported by the Natural Science Foundation of China (Grant Nos. 51773062 and 61831021). We thank Dr. Zhi Liu (Pasteur Institute, Chinese Academy of Science, China) for Cryo-TEM characterization and Dr. Sheng Yang (Max Planck Institute for Solid State Research, Germany) for helpful discussion, and also thank the Material structure analysis center and Multifunctional Platform for Innovation of East China Normal University (003, 006), and the Center for Advanced Electronic Materials and Devices (AEMD) of Shanghai Jiao Tong University.

Open Access This article is licensed under a Creative Commons Attribution 4.0 International License, which permits use, sharing, adaptation, distribution and reproduction in any medium or format, as long as you give appropriate credit to the original author(s) and the source, provide a link to the Creative Commons licence, and indicate if changes were made. The images or other third party material in this article are included in the article's Creative Commons licence, unless indicated otherwise in a credit line to the material. If material is not included in the article's Creative Commons licence and your intended use is not permitted by statutory regulation or exceeds the permitted use, you will need to obtain permission directly from the copyright holder. To view a copy of this licence, visit http://creativecommons.org/licenses/by/4.0/.

Electronic supplementary material The online version of this article (https://doi.org/10.1007/s40820-020-0370-1) contains supplementary material, which is available to authorized users.

\section{References}

1. K. Biswas, J. He, I.D. Blum, C.-I. Wu, T.P. Hogan, D.N. Seidman, V.P. Dravid, M.G. Kanatzidis, High-performance bulk thermoelectrics with all-scale hierarchical architectures. Nature 489, 414-418 (2012). https://doi.org/10.1038/natur e11439

2. R. Mezzenga, J.M. Seddon, C.J. Drummond, B.J. Boyd, G.E. Schröder-Turk, L. Sagalowicz, Nature-inspired design and application of lipidic lyotropic liquid crystals. Adv. Mater. (2019). https://doi.org/10.1002/adma.201900818

3. K. Chung, S. Yu, C.-J. Heo, J.W. Shim, S.-M. Yang et al., Flexible, angle-independent, structural color reflectors inspired by morpho butterfly wings. Adv. Mater. 24(18), 2375-2379 (2012). https://doi.org/10.1002/adma.201200521

4. L.B. Gower, Biomimetic model systems for investigating the amorphous precursor pathway and its role in biomineralization. Chem. Rev. 108(11), 4551-4627 (2008). https://doi. org/10.1021/cr800443h

5. U.G. Wegst, H. Bai, E. Saiz, A.P. Tomsia, R.O. Ritchie, Bioinspired structural materials. Nat. Mater. 14(1), 23-36 (2015). https://doi.org/10.1038/nmat4089

6. R. Lakes, Materials with structural hierarchy. Nature 361(6412), 511-515 (1993). https://doi.org/10.1038/361511a0
7. Y. Zhang, B.Y.W. Hsu, C. Ren, X. Li, J. Wang, Silica-based nanocapsules: synthesis, structure control and biomedical applications. Chem. Soc. Rev. 44(1), 315-335 (2015). https ://doi.org/10.1039/C4CS00199K

8. X.-Y. Yang, L.-H. Chen, Y. Li, J.C. Rooke, C. Sanchez, B.-L. $\mathrm{Su}$, Hierarchically porous materials: synthesis strategies and structure design. Chem. Soc. Rev. 46(2), 481-558 (2017). https://doi.org/10.1039/C6CS00829A

9. A.H. Groschel, F.H. Schacher, H. Schmalz, O.V. Borisov, E.B. Zhulina, A. Walther, A.H. Muller, Precise hierarchical selfassembly of multicompartment micelles. Nat. Commun. 3, 710 (2012). https://doi.org/10.1038/ncomms1707

10. S. Park, J.-H. Lim, S.-W. Chung, C.A. Mirkin, Self-assembly of mesoscopic metal-polymer amphiphiles. Science 303(5656), 348 (2004). https://doi.org/10.1126/science.10932 76

11. G. Férey, F. Millange, M. Morcrette, C. Serre, M.-L. Doublet, J.-M. Grenèche, J.-M. Tarascon, Mixed-valence Li/Fe-based metal-organic frameworks with both reversible redox and sorption properties. Angew. Chem. Int. Ed. 46(18), 3259-3263 (2007). https://doi.org/10.1002/anie.200605163

12. M. Faustini, L. Nicole, E. Ruiz-Hitzky, C. Sanchez, History of organic-inorganic hybrid materials: prehistory, art, science, and advanced applications. Adv. Funct. Mater. 28(27), 1704158 (2018). https://doi.org/10.1002/adfm.201704158

13. M. Pramanik, Y. Tsujimoto, V. Malgras, S.X. Dou, J.H. Kim, Y. Yamauchi, Mesoporous iron phosphonate electrodes with crystalline frameworks for lithium-ion batteries. Chem. Mater. 27(3), 1082-1089 (2015). https://doi.org/10.1021/cm5044045

14. M.H. Sun, S.Z. Huang, L.H. Chen, Y. Li, X.Y. Yang, Z.Y. Yuan, B.L. Su, Applications of hierarchically structured porous materials from energy storage and conversion, catalysis, photocatalysis, adsorption, separation, and sensing to biomedicine. Chem. Soc. Rev. 45(12), 3479-3563 (2016). https ://doi.org/10.1039/c6cs00135a

15. K.P. Singh, E.J. Bae, J.S. Yu, Fe-P: a new class of electroactive catalyst for oxygen reduction reaction. J. Am. Chem. Soc. 137(9), 3165-3168 (2015). https://doi.org/10.1021/Ja511759u

16. J. Song, B. Zhou, H. Zhou, L. Wu, Q. Meng, Z. Liu, B. Han, Porous zirconium-phytic acid hybrid: a highly efficient catalyst for Meerwein-Ponndorf-Verley reductions. Angew. Chem. Int. Ed. 54(32), 9399-9403 (2015). https://doi.org/10.1002/ anie. 201504001

17. L. Li, G. Zhang, Z. Su, One-step assembly of phytic acid metal complexes for superhydrophilic coatings. Angew. Chem. Int. Ed. 55(31), 9093-9096 (2016). https://doi.org/10.1002/ anie. 201604671

18. H. Zhou, X. Li, Y. Li, M. Zheng, H. Pang, Applications of $\mathrm{M}_{\mathrm{x}} \mathrm{Se}_{\mathrm{y}}(\mathrm{M}=\mathrm{Fe} \mathrm{Co}, \mathrm{Ni})$ and their composites in electrochemical energy storage and conversion. Nano-Micro Lett. 11, 40 (2019). https://doi.org/10.1007/s40820-019-0272-2

19. N. Zhang, X. Han, Y. Liu, X. Hu, Q. Zhao, J. Chen, 3D porous $\gamma-\mathrm{Fe}_{2} \mathrm{O}_{3} @ \mathrm{C}$ nanocomposite as high-performance anode material of Na-ion batteries. Adv. Energy Mater. 5(5), 1401123 (2015). https://doi.org/10.1002/aenm.201401123 
20. J. Zhang, S. Karmakar, M. Yu, N. Mitter, J. Zou, C. Yu, Synthesis of silica vesicles with controlled entrance size for high loading, sustained release, and cellular delivery of therapeutical proteins. Small 10(24), 5068-5076 (2014). https://doi. org/10.1002/smll.201401538

21. H. Wang, X. Zhou, M. Yu, Y. Wang, L. Han et al., Supraassembly of siliceous vesicles. J. Am. Chem. Soc. 128(50), 15992-15993 (2006). https://doi.org/10.1021/ja066707o

22. D. Kong, J. Zoñ, J. McBee, A. Clearfield, Rational design and synthesis of porous organic-inorganic hybrid frameworks constructed by 1,3,5-benzenetriphosphonic acid and pyridine synthons. Inorg. Chem. 45(3), 977-986 (2006). https://doi. org/10.1021/ic0509377

23. F. Yang, H. Gao, J. Hao, S. Zhang, P. Li, Y. Liu, J. Chen, Z. Guo, Yolk-shell structured FeP@C nanoboxes as advanced anode materials for rechargeable lithium-/potassium-ion batteries. Adv. Funct. Mater. 29(16), 1808291 (2019). https://doi. org/10.1002/adfm.201808291

24. H. Kunieda, K. Shinoda, Krafft points, critical micelle concentrations, surface tension, and solubilizing power of aqueous solutions of fluorinated surfactants. J. Phys. Chem. C 80(22), 2468-2470 (1976). https://doi.org/10.1021/j100563a007

25. J. Zhang, A. Song, Z. Li, G. Xu, J. Hao, Phase behaviors and self-assembly properties of two catanionic surfactant systems: $\mathrm{C}_{8} \mathrm{~F}_{17} \mathrm{COOH} / \mathrm{tTaOH} / \mathrm{H}_{2} \mathrm{O}$ and $\mathrm{C}_{8} \mathrm{H}_{17} \mathrm{COOH} / \mathrm{tTaOH} / \mathrm{H}_{2} \mathrm{O}$. J. Phys. Chem. B 114(41), 13128-13135 (2010). https://doi. org/10.1021/jp104579h

26. J. Wei, G. Wang, F. Chen, M. Bai, Y. Liang, H. Wang, D. Zhao, Y. Zhao, Sol-gel synthesis of metal-phenolic coordination spheres and their derived carbon composites. Angew. Chem. Int. Ed. 57(31), 9838-9843 (2018). https://doi.org/10.1002/ anie. 201805781

27. S. Liu, F. Wang, R. Dong, T. Zhang, J. Zhang, X. Zhuang, Y. Mai, X. Feng, Dual-template synthesis of 2D mesoporous polypyrrole nanosheets with controlled pore size. Adv. Mater. 28(38), 8365-8370 (2016). https://doi.org/10.1002/ adma.201603036

28. S. Liu, J. Zhang, R. Dong, P. Gordiichuk, T. Zhang et al., Two-dimensional mesoscale-ordered conducting polymers. Angew. Chem. Int. Ed. 55(40), 12516-12521 (2016). https:// doi.org/10.1002/anie.201606988

29. Y. Wen, F. Wei, W. Zhang, A. Cui, J. Cui et al., Two-dimensional mesoporous sensing materials. Chin. Chem. Lett. (2019). https://doi.org/10.1016/j.cclet.2019.04.071. (in press)

30. Y. Wang, X. Yu, S. Xu, J. Bai, R. Xiao et al., A zero-strain layered metal oxide as the negative electrode for long-life sodium-ion batteries. Nat. Commun. 4, 2365 (2013). https:// doi.org/10.1038/ncomms3365

31. W. Guo, W. Sun, L.-P. Lv, S. Kong, Y. Wang, Microwaveassisted morphology evolution of Fe-based metal-organic frameworks and their derived $\mathrm{Fe}_{2} \mathrm{O}_{3}$ nanostructures for $\mathrm{Li}$ ion storage. ACS Nano 11(4), 4198-4205 (2017). https://doi. org/10.1021/acsnano.7b01152
32. Y. Yan, Y.-X. Yin, Y.-G. Guo, L.-J. Wan, A sandwich-like hierarchically porous carbon/graphene composite as a highperformance anode material for sodium-ion batteries. Adv. Energy Mater. 4(8), 1301584 (2014). https://doi.org/10.1002/ aenm.201301584

33. N. Yabuuchi, K. Kubota, M. Dahbi, S. Komaba, Research development on sodium-ion batteries. Chem. Rev. 114(23), 11636-11682 (2014). https://doi.org/10.1021/cr500192f

34. J.-Y. Hwang, S.-T. Myung, Y.-K. Sun, Sodium-ion batteries: present and future. Chem. Soc. Rev. 46(12), 3529-3614 (2017). https://doi.org/10.1039/C6CS00776G

35. Y. Fang, L. Xiao, J. Qian, X. Ai, H. Yang, Y. Cao, Mesoporous amorphous $\mathrm{FePO}_{4}$ nanospheres as high-performance cathode material for sodium-ion batteries. Nano Lett. 14(6), 35393543 (2014). https://doi.org/10.1021/n1501152f

36. K. Lan, Y. Liu, W. Zhang, Y. Liu, A. Elzatahry et al., Uniform ordered two-dimensional mesoporous $\mathrm{TiO}_{2}$ nanosheets from hydrothermal-induced solvent-confined monomicelle assembly. J. Am. Chem. Soc. 140(11), 4135-4143 (2018). https:// doi.org/10.1021/jacs.8b00909

37. H. Liu, W. Li, D. Shen, D. Zhao, G. Wang, Graphitic carbon conformal coating of mesoporous $\mathrm{TiO}_{2}$ hollow spheres for high-performance lithium ion battery anodes. J. Am. Chem. Soc. 137(40), 13161-13166 (2015). https://doi.org/10.1021/ jacs.5b08743

38. P. Mei, J. Lee, M. Pramanik, A. Alshehri, J. Kim et al., Mesoporous manganese phosphonate nanorods as a prospective anode for lithium-ion batteries. ACS Appl. Mater. Interfaces. 10(23), 19739-19745 (2018). https://doi.org/10.1021/ acsami.8b05292

39. D. Zhao, T. Meng, J. Qin, W. Wang, Z. Yin, M. Cao, Rational construction of multivoids-assembled hybrid nanospheres based on $\mathrm{VPO}_{4}$ encapsulated in porous carbon with superior lithium storage performance. ACS Appl. Mater. Interfaces. 9(2), 1437-1445 (2017). https://doi.org/10.1021/acsami.6b116 70

40. Z. Xu, J. Yang, T. Zhang, Y. Nuli, J. Wang, S.-I. Hirano, Silicon microparticle anodes with self-healing multiple network binder. Joule 2(5), 950-961 (2018). https://doi.org/10.1016/j. joule.2018.02.012

41. H. Ying, W.-Q. Han, Metallic Sn-based anode materials: application in high-performance lithium-ion and sodium-ion batteries. Adv. Sci. 4(11), 1700298 (2017). https://doi.org/10.1002/ advs.201700298

42. W. Wang, J. Zhou, Z. Wang, L. Zhao, P. Li et al., Short-range order in mesoporous carbon boosts potassium-ion battery performance. Adv. Energy Mater. 8(5), 1701648 (2018). https:// doi.org/10.1002/aenm.201701648

43. K. Lan, Y. Xia, R. Wang, Z. Zhao, W. Zhang et al., Confined interfacial monomicelle assembly for precisely controlled coating of single-layered titania mesopores. Matter (2019). https://doi.org/10.1016/j.matt.2019.03.003 